\section{(1)}

CrossMark

\title{
Relationship of obstructive sleep apnoea severity and subclinical systemic atherosclerosis
}

\author{
Soriul Kim ${ }^{1}$, Ki Yeol Lee ${ }^{2,6}$, Nan Hee Kim³ ${ }^{3}$ Robert D. Abbott ${ }^{1}$, Cherry Kim² $^{2}$, \\ Seung Ku Lee (10) ${ }^{1}$, Seong Hwan Kim $\mathbb{1}^{4}$ and Chol Shin ${ }^{1,5,6}$
}

Affiliations: ${ }^{1}$ Institute for Human Genomic Study, College of Medicine, Korea University, Seoul, Republic of Korea. ${ }^{2}$ Dept of Radiology, Korea University Ansan Hospital, Ansan, Republic of Korea. ${ }^{3}$ Division of Endocrinology and Metabolism, Dept of Internal Medicine, Korea University Ansan Hospital, Ansan, Republic of Korea. ${ }^{4}$ Division of Cardiology, Dept of Internal Medicine, Korea University Ansan Hospital, Ansan, Republic of Korea. ${ }^{5}$ Division of Pulmonary Sleep and Critical Care Medicine, Dept of Internal Medicine, Korea University Ansan Hospital, Ansan, Republic of Korea. ${ }^{6}$ These two authors contributed equally to this work.

Correspondence: Chol Shin, Division of Respiratory and Critical Care, Dept of Internal Medicine, Korea University Ansan Hospital, \#123, Jeokgeum-ro, Danwon-gu, Ansan-si, Gyeonggi-do 15355, Republic of Korea. E-mail: chol-shinakorea.ac.kr

@ERSpublications

In this large population-based study, obstructive sleep apnoea was associated with greater thoracic aorta calcification as early signs of cardiovascular disease, especially in patients with higher epicardial fat volume http://bit.ly/31vljKV

Cite this article as: Kim S, Lee KY, Kim NH, et al. Relationship of obstructive sleep apnoea severity and subclinical systemic atherosclerosis. Eur Respir J 2020; 55: 1900959 [https://doi.org/10.1183/ 13993003.00959-2019].

ABSTRACT Obstructive sleep apnoea (OSA) is a common form of sleep disordered breathing. Untreated OSA might accelerate atherosclerosis, potentially increasing the cardiovascular disease burden in patients. The present study aimed to evaluate the association between objectively measured OSA severity and the presence of subclinical systemic atherosclerosis using noninvasive measurements, including tomographic quantification of the calcium burden.

A total of 2157 participants of the Korean Genome and Epidemiology Study, who were free of structural heart disease and underwent both in-home polysomnography and chest computed tomography, were cross-sectionally analysed. Participants were divided into three groups based on the severity of OSA: no

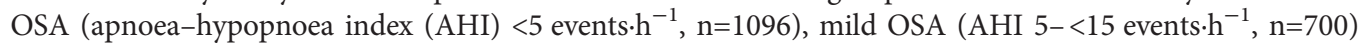
and moderate-to-severe OSA (AHI $\geqslant 15$ events $\cdot \mathrm{h}^{-1}, \mathrm{n}=361$ ). Calcium deposits in the thoracic aorta and coronary arteries were measured by the Agatston score.

Participants with moderate-to-severe OSA were 1.6 times (95\% CI 1.18-2.15 times; $\mathrm{p}=0.002$ ) more likely to have ascending thoracic aorta calcification ( $\geqslant 100$ units) than those without OSA, after adjustment for cardiovascular risk factors. In addition, the association between moderate-to-severe OSA and ascending thoracic aorta calcification of subjects with higher epicardial fat volume was slightly stronger than that in patients without OSA and in the lowest epicardial fat volume tertile (OR 2.11, 95\% CI 1.30-3.43).

Severity of OSA in the general population was independently associated with subclinical systemic atherosclerosis. These findings highlight the potential importance of severe OSA, especially in subjects with higher epicardial fat, as a possible predictive factor for systemic atherosclerosis and cardiovascular disease. 


\section{Introduction}

Obstructive sleep apnoea (OSA) is a common form of sleep disordered breathing characterised by repeated partial or complete obstruction of the upper airway, which leads to intermittent hypoxia, intrathoracic pressure changes and sleep fragmentation $[1,2]$. The reported prevalence of OSA in the Asian population using sleep monitoring ranges from $9 \%$ to $89 \%$ (mild OSA from $30 \%$ to $89 \%$ and moderate-to-severe OSA from $9 \%$ to $51 \%$ ). The overall prevalence of OSA in South Korea, ascertained using sleep questionnaires, was reported to be $15 \%[3,4]$. Although the measurement techniques and scoring criteria for OSA have changed over several decades, the awareness and knowledge of OSA among the general population is still poor. According to a population-based study, about one in five participants was aware of OSA and only one in 10 participants was able to define OSA correctly [5]. Unrecognised and untreated OSA was associated with increased healthcare utilisation and cost, as well as a higher comorbid diseases burden [6]. OSA can accelerate hypertension, diabetes, atherosclerosis, stroke and cardiovascular disease (CVD), potentially increasing cardiovascular mortality in patients with OSA [7-9]. Frequently occurring and coexisting cardio-metabolic disorders make it difficult to assess the independent effect of OSA on the progression of atherosclerosis as well as CVD [10].

Scoring the amount of calcium in the aorta and coronary arteries using cardiac and chest computed tomography (CT) provides a noninvasive measure of the subclinical atherosclerotic burden. Increased calcification in both the aorta and coronary arteries has been established as a distinct marker of underlying atherosclerosis in the vascular beds, and has been identified to provide further prognostic information on CVD [11-13]. Moreover, accumulating evidence has linked sleep disturbances to increased coronary artery calcification (CAC) and inflammation may represent an important factor with respect to OSA-induced atherosclerotic calcification; however, the results of these studies have been inconsistent [13-17]. Some results have revealed the association to be nonsignificant after adjustment for cardiovascular risk factors, such as body mass index (BMI) [16]. These inconsistencies illustrate the complexity of the interaction between sleep disordered breathing and atherosclerotic calcification, as well as the multifactorial involvement of increased cardiovascular risk associated with OSA.

In addition, carotid intima-media thickness (cIMT) and pulse wave velocity (PWV) by noninvasive measurements are early indicators of subclinical atherosclerosis that predict future CVD events [18]. In systematic and meta-analysis studies, patients with OSA had significantly higher cIMT and PWVs, suggestive of an atherosclerotic process, but there was significant heterogeneity [19]. In other words, various cardiovascular risk factors have been associated with an increase in cIMT and PWV, and OSA is one such potential risk factor. In addition, increased atherosclerosis and vascular stiffness may occur with increased severity of OSA in asymptomatic CVD.

The present study aimed to evaluate the association between objectively measured OSA severity and the presence of subclinical systemic atherosclerosis using noninvasive measurements, including tomographic quantification of the calcium burden in the aorta and coronary arteries in healthy individuals without structural heart disease. Furthermore, we examined whether epicardial fat volume (EFV) as an unambiguous cardiovascular risk factor has a moderating effect on the relationship between OSA severity and the presence of subclinical systemic atherosclerosis.

\section{Material and methods \\ Study design and population}

This cross-sectional study was performed from a population-based cohort comprised of Korean males and females aged 39-70 years. All subjects were participants between 2001 and 2002 in the Korean Genome and Epidemiology Study (KoGES), which is an ongoing prospective investigation. Detailed information on participant recruitment is available [20,21]. Briefly, a total of 5012 participants from Ansan, Republic of Korea, were examined at baseline from 2001 to 2002. Cohort members completed a comprehensive health examination and questionnaire-based interview, and biospecimens for assays were collected by health professionals. The health examination was comprised of anthropometric and clinical evaluations, including chest radiography. The questionnaire covered demographic characteristics, lifestyle and medical history. Follow-up examinations were performed biennially during a scheduled site visit. The eighth examination was performed between March 2015 and December 2016, and was attended by 3083 individuals. Of these participants, 2378 underwent overnight in-home polysomnography (PSG) and chest CT for the measurement of the calcification of the thoracic aorta and coronary arteries and EFV measurement. From this sample, we further excluded participants with prevalent myocardial infarction, angina pectoris, congenital heart failure, arrhythmia or stroke $(n=221)$. The final data were analysed from 2157 individuals (figure 1).

Written informed consent was obtained from all participants at every site visit. The study protocol was approved by the Institutional Ethics Committee of Korea University Ansan Hospital (Ansan). 


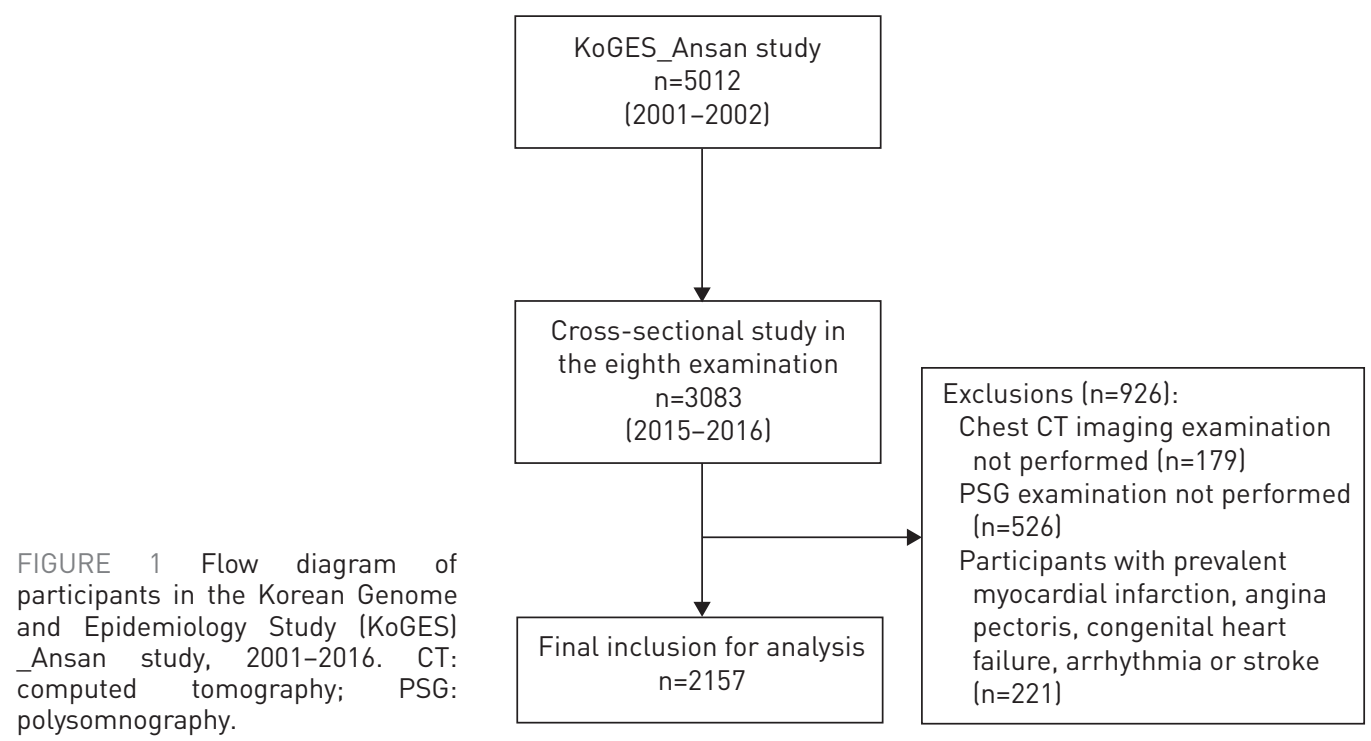

\section{Polysomnography}

Overnight in-home PSG was performed using a portable monitoring device (Embletta X-100; Embla Systems, Broomfield, CO, USA) with channels for electroencephalography, ECG, a pressure transducer airflow sensor, a chest and abdominal respiratory movement sensor, and a snore sensor. A trained technician connected the device to the patient at bedtime, data were collected the morning after the unattended overnight recording and the PSG results were manually scored according to standard criteria [22]. An oxygen desaturation event was detected when the oxygen saturation dropped by at least $4 \%$. All saturations values $<50 \%$ were excluded as artefact values and not counted as part of a desaturation event. An apnoea event was detected if both of the following criteria were met: 1) there was a drop in the peak signal excursion by $\geqslant 90 \%$ of the pre-event baseline (reference amplitude) and 2) the duration of the $\geqslant 90 \%$ drop in sensor signal was $\geqslant 10 \mathrm{~s}$. In addition, a hypopnoea event was detected if all of the following criteria were met: 1) the peak signal excursions dropped by $\geqslant 30 \%$ of the reference amplitude, 2) the duration of the $\geqslant 30 \%$ drop in signal excursions was $\geqslant 10 \mathrm{~s}$ and 3 ) there was $\geqslant 3 \%$ oxygen desaturation from the reference amplitude or the event was associated with an arousal. The reference amplitude was calculated as the mean value of the peak amplitudes in the period of $100 \mathrm{~s}$ preceding the event. The apnoea-hypopnoea index (AHI) was calculated by averaging the total number of obstructive apnoea and hypopnoea events per hour of sleep, and OSA severity was defined according to different levels of AHI: no OSA (AHI $<5$ events $\cdot \mathrm{h}^{-1}$ ), mild OSA (AHI $5-<15$ events $\cdot \mathrm{h}^{-1}$ ) and moderate-to-severe OSA (AHI $\geqslant 15$ events. $\mathrm{h}^{-1}$ ).

\section{Chest CT imaging protocol and measurements}

Details on the procedures for chest CT data acquisition, scanner quality assurance and scan reading have been previously described [23]. Briefly, well-trained technicians performed chest CT scans using a 64-multidetector CT scanner (Brilliance 64; Philips Healthcare, Cleveland, OH, USA) following a standardised protocol, with each participant in the supine position during end-inspiratory and end-expiratory breath-hold. Scanning parameters were held constant at the $64 \times 0.625 \mathrm{~mm}$ detector configuration, $120 \mathrm{kV}$ (peak), $100 \mathrm{mAs}$ and a section thickness of $0.625 \mathrm{~mm}$ without intravenous contrast material.

The presence of aortic calcification was defined as the presence of at least one detectable lesion. We quantified calcifications in the walls of the ascending and descending thoracic aorta from the aortic root (above the left main coronary artery) to the level of the diaphragmatic crura (below both the ventricles). All calcification scores were calculated using the Agatston scoring method [24].

EFV was measured three-dimensionally in all patients using noncontrast chest CT images [24, 25]. Epicardial fat was defined as fat enclosed in the visceral pericardium. Segmentation of the overall volume was automatically interpolated using manually defined tracings and EFV was subsequently quantified by calculating the total volume of the tissue in which CT density ranged from -500 to $0 \mathrm{HU}$ within the pericardial cavity.

Quantitative analysis of aortic calcification and EFV was performed using Aquarius iNtuition Edition version 4.4.11 (TeraRecon, Foster City, CA, USA), which is an automated lung image analysis tool. 
Common cIMT and PWV measurements

Measurements of cIMT on the right and left carotid arteries were performed using B-mode ultrasonography (Titan; Sonosite, Tokyo, Japan) with a $7.5 \mathrm{MHz}$ linear array transducer. For IMT measurements, images of the distal common carotid arteries were obtained at both the far and near walls, $\sim 1 \mathrm{~cm}$ proximal to the bulb. In each segment, the mean values of the common carotid arteries IMT (CCA-IMT) were calculated as the average of the left and right CCA-IMT scores obtained from the near and far walls using semiautomated software (M'ATH SR version 2.0; Metris, Argenteuil, France). Two technicians were trained with an authorised protocol and certified, and the intraclass correlation coefficient values obtained from them was $>0.90$ for each segment (range 0.910-0.941) [26].

The brachial-ankle PWV (baPWV) was measured in the supine position using an automatic volume-plethysmographic device (VP2000; Colin, Komaki, Japan). The PWV was defined as the distance of two sites over the pulse transit time. The time interval between the brachium and the ankle was defined as the time interval between the wave front of the brachial waveform and that of the ankle waveform. The distance between sampling points of the baPWV was calculated automatically according to the heights of the subjects [27].

\section{Other variables}

Demographic data, smoking status, amount of alcohol consumed, physical activity and medical conditions were obtained via a questionnaire. Physical activity was assessed using a scale consisting of five categories for activity intensity as measured by hours spent in a typical day per intensity level. The total metabolic equivalent $\left(\mathrm{MET} \cdot\right.$ week $^{-1}$ ) score was calculated by multiplying the hours spent at a particular activity intensity by the MET value. BMI was calculated as weight $(\mathrm{kg})$ divided by height squared $\left(\mathrm{m}^{2}\right)$. Blood samples were collected after a fasting period of at least $8 \mathrm{~h}$. Fasting glucose, haemoglobin A1c, total cholesterol, triglyceride, high-density lipoprotein (HDL)-cholesterol and high-sensitivity C-reactive protein (hsCRP) levels were measured using standardised enzymatic methods in a commercial laboratory (Seoul Clinical Laboratories, Seoul, Republic of Korea). Low-density lipoprotein (LDL)-cholesterol levels were estimated using the Friedewald formula. Hypertension was defined as a systolic blood pressure of $\geqslant 140 \mathrm{mmHg}$, a diastolic blood pressure of $\geqslant 90 \mathrm{mmHg}$ or the current use of antihypertensive medications. Type 2 diabetes was defined as a fasting blood glucose level of $\geqslant 126 \mathrm{mg} \cdot \mathrm{dL}^{-1}$ or the use of insulin or oral hypoglycaemic medications.

\section{Statistical analysis}

The general characteristics of participants are presented as mean with standard deviation and proportions, stratified by OSA severity (AHI $<5,5-<15$ and $\geqslant 15$ events $\mathrm{h}^{-1}$ ) using the Chi-squared test or one-way ANOVA for categorical and continuous variables, respectively. Because thoracic aorta calcification (TAC) and CAC scores had a highly positively skewed distribution, we used log-transformed values (log (raw score+1)) to deal with individuals who had raw scores of zero. The associations between the subclinical systemic atherosclerosis parameters and severity of OSA were determined using one-way ANCOVA and the post hoc Scheffe test. In the intervening time, subjects were classified into two groups according to the presence or absence of TAC or CAC using each raw Agatston score: the presence of ascending TAC (aTAC score $\geqslant 100$ units), descending TAC (dTAC score $\geqslant 100$ units), overall TAC (TAC score $\geqslant 400$ units) and CAC (CAC score $>0$ units). Multivariate logistic regression analysis was performed to determine the effects of subclinical systemic atherosclerosis. Model 1 was unadjusted, whereas model 2 was adjusted for age, sex and BMI. Additionally, model 3 was also adjusted for hypertension, type 2 diabetes, HDL-cholesterol, LDL-cholesterol and pack-years of smoking. Tests for moderate effects were examined to assess whether the associations between OSA and subclinical systemic atherosclerosis varied across EFV tertiles. All statistical analyses were performed using SAS version 9.4 (SAS Institute, Cary, NC, USA).

\section{Results}

\section{Descriptive analysis}

A total of 2157 participants (mean age $59.96 \pm 6.67$ years; $48.73 \%$ male) were examined. Of all the participants, $50.81 \%$ had no OSA (AHI $<5$ events $\cdot \mathrm{h}^{-1}$ ), $32.45 \%$ had mild OSA (AHI $5-<15$ events $^{-1} \mathrm{~h}^{-1}$ ) and $16.74 \%$ had moderate-to-severe OSA (AHI $\geqslant 15$ events $\left.\cdot \mathrm{h}^{-1}\right)$. Participants with moderate-to-severe OSA were slightly older $(62.08 \pm 7.63$ versus $58.76 \pm 5.96$ years; $\mathrm{p}<0.001)$, were more often male $(68.14 \%$ versus 41.61\%; $<<0.001)$, had a higher EFV $\left(285.24 \pm 69.69\right.$ versus $\left.234.13 \pm 59.97 \mathrm{~cm}^{3} ; \mathrm{p}<0.001\right)$, higher prevalence of hypertension $(62.60 \%$ versus $34.12 \% ; \mathrm{p}<0.001)$ and type 2 diabetes $(43.21 \%$ versus $25.64 \% ; \mathrm{p}<0.001)$, and had lower levels of HDL-cholesterol (43.09 \pm 10.82 versus $\left.48.20 \pm 12.06 \mathrm{mg} \cdot \mathrm{dL}^{-1} ; \mathrm{p}<0.001\right)$ than individuals without OSA. Interestingly, hsCRP levels and physical activity were not different among the OSA severities (table 1). 
TABLE 1 Comparison of clinical characteristics according to the severity of obstructive sleep apnoea (OSA)

\begin{tabular}{|c|c|c|c|c|}
\hline $\begin{array}{c}\text { No OSA } \\
\left(A H I<5 \text { events } \cdot h^{-1}\right)\end{array}$ & $\begin{array}{c}\text { Mild OSA } \\
\left(\text { AHI } 5-<15 \text { events } \cdot h^{-1}\right)\end{array}$ & $\begin{array}{l}\text { Moderate-to-severe OSA } \\
\left(A H I \geqslant 15 \text { events } \cdot h^{-1}\right)\end{array}$ & p-value ${ }^{\#}$ & p-value ${ }^{\pi}$ \\
\hline 1096 (50.8) & 700 (32.5) & 361 (16.7) & & \\
\hline $58.8 \pm 6.0$ & $60.8 \pm 6.8$ & $62.1 \pm 7.6$ & $<0.001$ & \\
\hline $456(41.6)$ & 349 (49.9) & $246(68.1)$ & $<0.001$ & \\
\hline $23.8 \pm 2.6$ & $25.2 \pm 2.9$ & $26.1 \pm 3.3$ & $<0.001$ & $<0.001^{+}$ \\
\hline $80.6 \pm 7.5$ & $85.2 \pm 8.2$ & $88.6 \pm 8.6$ & $<0.001$ & $<0.001^{+}$ \\
\hline $234.1 \pm 60.0$ & $260.3 \pm 66.8$ & $285.2 \pm 69.7$ & $<0.001$ & $<0.001^{+}$ \\
\hline $113.4 \pm 14.3$ & $116.0 \pm 13.4$ & $118.5 \pm 13.1$ & $<0.001$ & $0.002^{+}$ \\
\hline $73.4 \pm 8.9$ & $74.6 \pm 9.0$ & $76.7 \pm 9.2$ & $<0.001$ & $<0.001^{+}$ \\
\hline $94.9 \pm 19.4$ & $99.2 \pm 22.7$ & $102.7 \pm 22.3$ & $<0.001$ & $<0.001^{+}$ \\
\hline $5.7 \pm 0.7$ & $5.9 \pm 0.9$ & $6.0 \pm 0.8$ & $<0.001$ & $<0.001^{+}$ \\
\hline $197.9 \pm 36.3$ & $192.5 \pm 35.9$ & $189.6 \pm 35.5$ & $<0.001$ & 0.22 \\
\hline $129.6 \pm 80.4$ & $140.3 \pm 94.0$ & $154.7 \pm 91.2$ & $<0.001$ & $<0.001^{+}$ \\
\hline $48.2 \pm 12.1$ & $46.4 \pm 11.2$ & $43.1 \pm 10.8$ & $<0.001$ & $<0.001^{+}$ \\
\hline $124.5 \pm 32.7$ & $119.2 \pm 31.6$ & $116.6 \pm 32.3$ & $<0.001$ & 0.06 \\
\hline $1.3 \pm 2.3$ & $1.3 \pm 2.5$ & $1.5 \pm 3.7$ & 0.26 & 0.33 \\
\hline $7.9 \pm 15.3$ & $9.4 \pm 16.0$ & $13.7 \pm 19.2$ & $<0.001$ & 0.64 \\
\hline $7.4 \pm 18.9$ & $8.9 \pm 20.0$ & $11.1 \pm 22.8$ & 0.01 & 0.70 \\
\hline $798.2 \pm 988.7$ & $809.1 \pm 940.4$ & $896.6 \pm 1145.5$ & 0.26 & 0.79 \\
\hline $374(34.1)$ & $353(50.4)$ & $226(62.6)$ & $<0.001$ & $<0.001$ \\
\hline $281(25.6)$ & $227(32.4)$ & $156(43.2)$ & $<0.001$ & $<0.001$ \\
\hline $2.1 \pm 1.4$ & $8.7 \pm 2.7$ & $26.1 \pm 12.1$ & $<0.001$ & $<0.001^{+}$ \\
\hline $4.3 \pm 5.5$ & $17.5 \pm 12.0$ & $41.8 \pm 18.9$ & $<0.001$ & $<0.001^{+}$ \\
\hline $0.8 \pm 1.2$ & $2.8 \pm 2.9$ & $11.2 \pm 13.5$ & $<0.001$ & $<0.001^{+}$ \\
\hline $371.4 \pm 80.0$ & $365.9 \pm 76.7$ & $366.2 \pm 79.8$ & 0.28 & 0.70 \\
\hline $4.7 \pm 3.6$ & $5.3 \pm 3.3$ & $7.0 \pm 4.9$ & $<0.001$ & $<0.001^{+}$ \\
\hline $95.9 \pm 1.1$ & $95.2 \pm 1.2$ & $94.4 \pm 1.5$ & $<0.001$ & $<0.001^{+}$ \\
\hline $89.6 \pm 5.3$ & $85.1 \pm 4.7$ & $80.4 \pm 6.7$ & $<0.001$ & $<0.001^{+}$ \\
\hline $1.9 \pm 0.3$ & $7.9 \pm 2.9$ & $24.0 \pm 11.6$ & $<0.001$ & $<0.001^{+}$ \\
\hline $0.5 \pm 2.7$ & $2.6 \pm 4.6$ & $16.2 \pm 26.0$ & $<0.001$ & $<0.001^{+}$ \\
\hline $0.2 \pm 0.9$ & $0.8 \pm 3.2$ & $4.7 \pm 7.9$ & $<0.001$ & $<0.001^{+}$ \\
\hline
\end{tabular}

Data are presented as $\mathrm{n}(\%)$ or mean $\pm \mathrm{SD}$, unless otherwise stated. $\mathrm{AHI}$ : apnoea-hypopnoea index; BMI: body mass index; EFV: epicardial fat volume; HbA1c: haemoglobin A1c; HDL: high-density lipoprotein; LDL: low-density lipoprotein; hsCRP: high-sensitivity C-reactive protein; MET: metabolic equivalent; TST: total sleep time; $S_{a_{2}}$ : arterial oxygen saturation. ${ }^{\#}$ : $p$-values for unadjusted model; ${ }^{\text {I: }} p$-values for multivariate models including age and sex where applicable; ${ }^{+}: p<0.05$ among all comparisons by the post hoc Scheffe test.

Comparisons of polysomnographic parameters and OSA severity are reported in table 1 . Supine AHI $\left(4.26 \pm 5.49\right.$ versus $41.75 \pm 18.87$ events $\left.\cdot \mathrm{h}^{-1}\right)$, nonsupine AHI $\left(0.80 \pm 1.16\right.$ versus $11.24 \pm 13.50$ events $\left.\cdot \mathrm{h}^{-1}\right)$ and oxygen desaturation index $\left(1.89 \pm 0.32\right.$ versus $24.04 \pm 11.62$ events $\left.\cdot \mathrm{h}^{-1}\right)$ as well as total AHI $(2.05 \pm 1.40$ versus $26.12 \pm 12.11$ events $\cdot \mathrm{h}^{-1}$ ) were higher in the moderate-to-severe OSA group than in the no OSA group (all adjusted $\mathrm{p}<0.001)$. Total sleep time was not different according to OSA severity; however, the awakening index of $>30 \mathrm{~s} \cdot \mathrm{h}^{-1}$ was greater in the moderate-to-severe OSA group than in the no OSA group $(4.67 \pm 3.63$ versus $6.96 \pm 4.85$ events. $\mathrm{h}^{-1}$; adjusted $\left.\mathrm{p}<0.001\right)$.

\section{Severity of OSA and subclinical systemic atherosclerosis}

Comparisons of measurements for subclinical systemic atherosclerosis parameters among OSA severities are reported in table 2. After adjusting for cardiovascular risk factors, baPWV and ankle-brachial index $(\mathrm{ABI})$ on the right ankle were increased in the moderate-to-severe OSA patients compared with individuals without OSA (baPWV: $1437.76 \pm 234.70$ versus $1569.14 \pm 321.18$; ABI: $1.167 \pm 0.076$ versus $1.182 \pm 0.087$; all $\mathrm{p}<0.05)$.

The median aTAC score was significantly higher in mild OSA (3.2 units) and moderate-to-severe OSA individuals (50.0 units) than in those without OSA ( 0.0 units) ( $\left.p_{\text {trend }}=0.02\right)$. The associations of dTAC and TAC with OSA severity were similar to the trend of aTAC ( $p_{\text {trend }}=0.008$ and 0.006 , respectively) (table 2 and figure 2). Moderate-to-severe OSA was associated with aTAC ( $\geqslant 100$ units) in the univariate logistic regression (OR 2.70, 95\% CI 2.10-3.47; p<0.001). In multivariate analyses, moderate-to-severe OSA remained independently associated with aTAC after adjusting for age, sex, BMI, hypertension, type 2 
TABLE 2 Associations with subclinical systemic atherosclerosis parameters and obstructive sleep apnoea (OSA) severity

\begin{tabular}{|c|c|c|c|}
\hline $\begin{array}{c}\text { No OSA } \\
\left(A H I<5 \text { events } \cdot h^{-1}\right)\end{array}$ & $\begin{array}{c}\text { Mild OSA } \\
\left(A H I 5-<15 \text { events } \cdot h^{-1}\right)\end{array}$ & $\begin{array}{c}\text { Moderate-to-severe 0SA } \\
\left(A H I \geqslant 15 \text { events } \cdot h^{-1}\right)\end{array}$ & p-value ${ }^{\#}$ \\
\hline $1096(50.81)$ & 700 (32.45) & $361(16.74)$ & \\
\hline $0.730 \pm 0.083$ & $0.741 \pm 0.082$ & $0.762 \pm 0.085$ & 0.24 \\
\hline $0.726 \pm 0.082$ & $0.745 \pm 0.077$ & $0.755 \pm 0.085$ & 0.75 \\
\hline $0.729 \pm 0.072$ & $0.743 \pm 0.069$ & $0.759 \pm 0.074$ & 0.86 \\
\hline $1437.76 \pm 234.70$ & $1495.38 \pm 247.07$ & $1569.14 \pm 321.18$ & $0.05^{\eta_{1,+}, \S}$ \\
\hline $1433.40 \pm 233.71$ & $1487.78 \pm 246.73$ & $1555.17 \pm 288.73$ & 0.14 \\
\hline $1.167 \pm 0.076$ & $1.180 \pm 0.066$ & $1.182 \pm 0.087$ & $0.003^{\text {ๆी,+ }}$ \\
\hline $1.156 \pm 0.071$ & $1.169 \pm 0.064$ & $1.167 \pm 0.072$ & $0.007^{\uparrow}$ \\
\hline $0.0(0.0-5782.8)$ & $3.2(0.0-4955.9)$ & $50.0(0.0-20749.0)$ & $0.02^{\text {१,+, }}$ \\
\hline $11.2(0.0-26255.3)$ & $50.9(0.0-21430.5)$ & $108.0(0.0-55353.6)$ & $0.008^{\|,+}, \S$ \\
\hline $37.9(0.0-27204.0)$ & $123.6(0.0-22167.0)$ & $265.5(0.0-59565.7)$ & $0.006^{\eta++, \S}$ \\
\hline $0.0(0.0-3039.4)$ & $0.0(0.0-1583.4)$ & $0.0(0.0-1808.6)$ & 0.29 \\
\hline
\end{tabular}

Data are presented as $\mathrm{n}(\%)$, mean \pm SD or median (range), unless otherwise stated. AHI: apnoea-hypopnoea index; IMT: intima-media thickness; PWV: pulse wave velocity; TAC: thoracic aorta calcification; CAC: coronary artery calcification. \#: p-values for one-way ANCOVA including age, sex, body mass index, hypertension, type 2 diabetes, high-density lipoprotein cholesterol, how-density lipoprotein cholesterol and pack-years of smoking; ๆ: $p<0.05$ when comparing no OSA to mild OSA by the post hoc Scheffe test; ${ }^{+}$: $p<0.05$ when comparing no OSA to moderate-to-severe OSA by the post hoc Scheffe test; ${ }^{\S}: \mathrm{p}<0.05$ when comparing mild OSA to moderate-to-severe OSA by the post hoc Scheffe test.

diabetes, HDL-cholesterol, LDL-cholesterol and pack-years of smoking (OR 1.59, 95\% CI 1.18-2.15; $\mathrm{p}=0.002)$ (table 3$)$. Both dTAC ( $\geqslant 100$ units) and TAC ( $\geqslant 400$ units) also had a positive correlation with moderate-to-severe OSA (OR 1.52, 95\% CI 1.12-2.07; $\mathrm{p}=0.007$ and OR 1.51, 95\% CI 1.10-2.07; $\mathrm{p}=0.01$, respectively). Based on unadjusted logistic regression, moderate-to-severe OSA was associated with CAC ( $>0$ units) (OR 1.96, 95\% CI 1.51-2.53; $\mathrm{p}<0.001$ ), but the relationship was not significant after adjusting for cardiovascular risk factors (OR 1.05, 95\% CI 0.78-1.42; $\mathrm{p}=0.75$ ).

Joint effects of OSA severity and EFV in subclinical systemic atherosclerosis

As shown in table 4, participants with moderate-to-severe OSA were 1.14 times (95\% CI 0.76-1.72 times) more likely to have aTAC ( $\geqslant 100$ units) relative to participants without OSA among participants in the

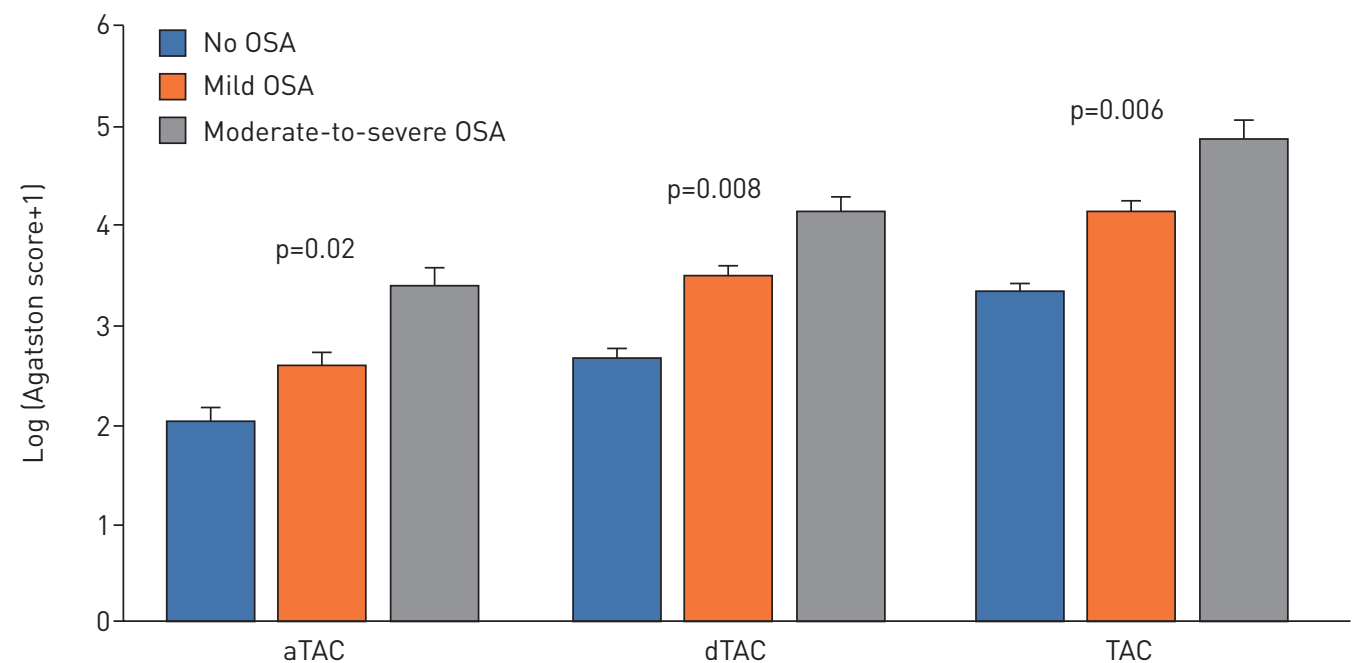

FIGURE 2 Severity of obstructive sleep apnoea (OSA) and thoracic aorta calcifications (TACs): ascending TAC (aTAC), descending TAC (dTAC) and TAC. TACs, which are useful predictors of subclinical systemic atherosclerosis, increased according to OSA severity (all adjusted $p<0.001$ using ANCOVA). All $p$-values are adjusted by age, sex, body mass index, hypertension, type 2 diabetes, high-density lipoprotein cholesterol, low-density lipoprotein cholesterol and pack-years of smoking. Error bars represent standard error. 
TABLE 3 Multivariate logistic regression analyses of the relationship between obstructive sleep apnoea (OSA) severity and the presence of thoracic aorta calcification (TAC) and coronary artery calcification (CAC)

\begin{tabular}{|c|c|c|c|c|c|c|}
\hline & \multirow[t]{2}{*}{$\begin{array}{c}\text { No OSA } \\
\left(A H I<5 \text { events } \cdot h^{-1}\right)\end{array}$} & \multicolumn{2}{|c|}{$\begin{array}{c}\text { Mild OSA } \\
\left(A H I 5-<15 \text { events } \cdot h^{-1}\right)\end{array}$} & \multicolumn{2}{|c|}{$\begin{array}{c}\text { Moderate-to-severe OSA } \\
\left(A H I \geqslant 15 \text { events } \cdot h^{-1}\right)\end{array}$} & \multirow[t]{2}{*}{$\mathbf{p}_{\text {trend }}$} \\
\hline & & OR $(95 \% \mathrm{CI})$ & $p$-value & OR $(95 \% \mathrm{CI})$ & p-value & \\
\hline Subjects & $1096(50.81)$ & \multirow{2}{*}{\multicolumn{2}{|c|}{700 (32.45) }} & \multirow{2}{*}{\multicolumn{2}{|c|}{$361(16.74)$}} & \\
\hline aTAC ( $\geqslant 100$ units $)$ & & & & & & \\
\hline Subjects & $260(23.72)$ & \multicolumn{2}{|l|}{229 (32.71) } & \multicolumn{2}{|c|}{165 (45.71) } & \\
\hline Model 1 & Reference & $1.57(1.27-1.93)$ & $<0.001$ & $2.70(2.10-3.47)$ & $<0.001$ & $<0.001$ \\
\hline Model 2 & Reference & $1.20(0.95-1.51)$ & 0.13 & $1.74(1.31-2.33)$ & $<0.001$ & $<0.001$ \\
\hline Model 3 & Reference & $1.14(0.90-1.46)$ & 0.27 & $1.59(1.18-2.15)$ & 0.002 & 0.003 \\
\hline \multicolumn{7}{|l|}{ dTAC ( $\geqslant 100$ units $)$} \\
\hline Subjects & $308(28.10)$ & \multicolumn{2}{|l|}{$291(41.57)$} & \multicolumn{2}{|c|}{178 (49.31) } & \\
\hline Model 1 & Reference & $1.81(1.48-2.21)$ & $<0.001$ & $2.54(1.98-3.25)$ & $<0.001$ & $<0.001$ \\
\hline Model 2 & Reference & $1.40(1.11-1.76)$ & 0.004 & $1.67(1.24-2.25)$ & $<0.001$ & $<0.001$ \\
\hline Model 3 & Reference & $1.35(1.07-1.71)$ & 0.01 & $1.52(1.12-2.07)$ & 0.007 & 0.003 \\
\hline \multicolumn{7}{|l|}{ TAC ( $\geqslant 400$ units $)$} \\
\hline Subjects & 235 (21.44) & \multicolumn{2}{|l|}{$231(33.00)$} & \multicolumn{2}{|c|}{157 (43.49) } & \\
\hline Model 1 & Reference & $1.81(1.46-2.23)$ & $<0.001$ & $2.82(2.19-3.63)$ & $<0.001$ & $<0.001$ \\
\hline Model 2 & Reference & $1.31(1.03-1.67)$ & 0.03 & $1.61(1.19-2.19)$ & 0.002 & 0.001 \\
\hline Model 3 & Reference & $1.25(0.97-1.61)$ & 0.09 & $1.51(1.10-2.07)$ & 0.01 & 0.008 \\
\hline \multicolumn{7}{|l|}{ CAC (>0 units) } \\
\hline Subjects & 248 (22.63) & \multicolumn{2}{|c|}{$181(25.86)$} & \multicolumn{2}{|c|}{131 (36.29) } & \\
\hline Model 1 & Reference & $1.19(0.96-1.49)$ & 0.12 & $1.96(1.51-2.53)$ & $<0.001$ & $<0.001$ \\
\hline Model 2 & Reference & $0.91(0.72-1.16)$ & 0.44 & $1.16(0.86-1.55)$ & 0.33 & 0.51 \\
\hline Model 3 & Reference & $0.84(0.66-1.08)$ & 0.18 & $1.05(0.78-1.42)$ & 0.75 & 0.98 \\
\hline
\end{tabular}

Data for numbers of subjects are presented as $n(\%)$. AHI: apnoea-hypopnoea index; aTAC: ascending TAC; dTAC: descending TAC. Model 1: unadjusted. Model 2: adjusted for age, sex and body mass index. Model 3 : adjusted model 2, hypertension, type 2 diabetes, high-density lipoprotein cholesterol, how-density lipoprotein cholesterol and pack-years of smoking.

TABLE 4 Multivariate logistic regression analysis of the presence of thoracic aorta calcification (TAC) and coronary artery calcification (CAC) for moderation effect of obstructive sleep apnoea (OSA) severity and epicardial fat volume (EFV)

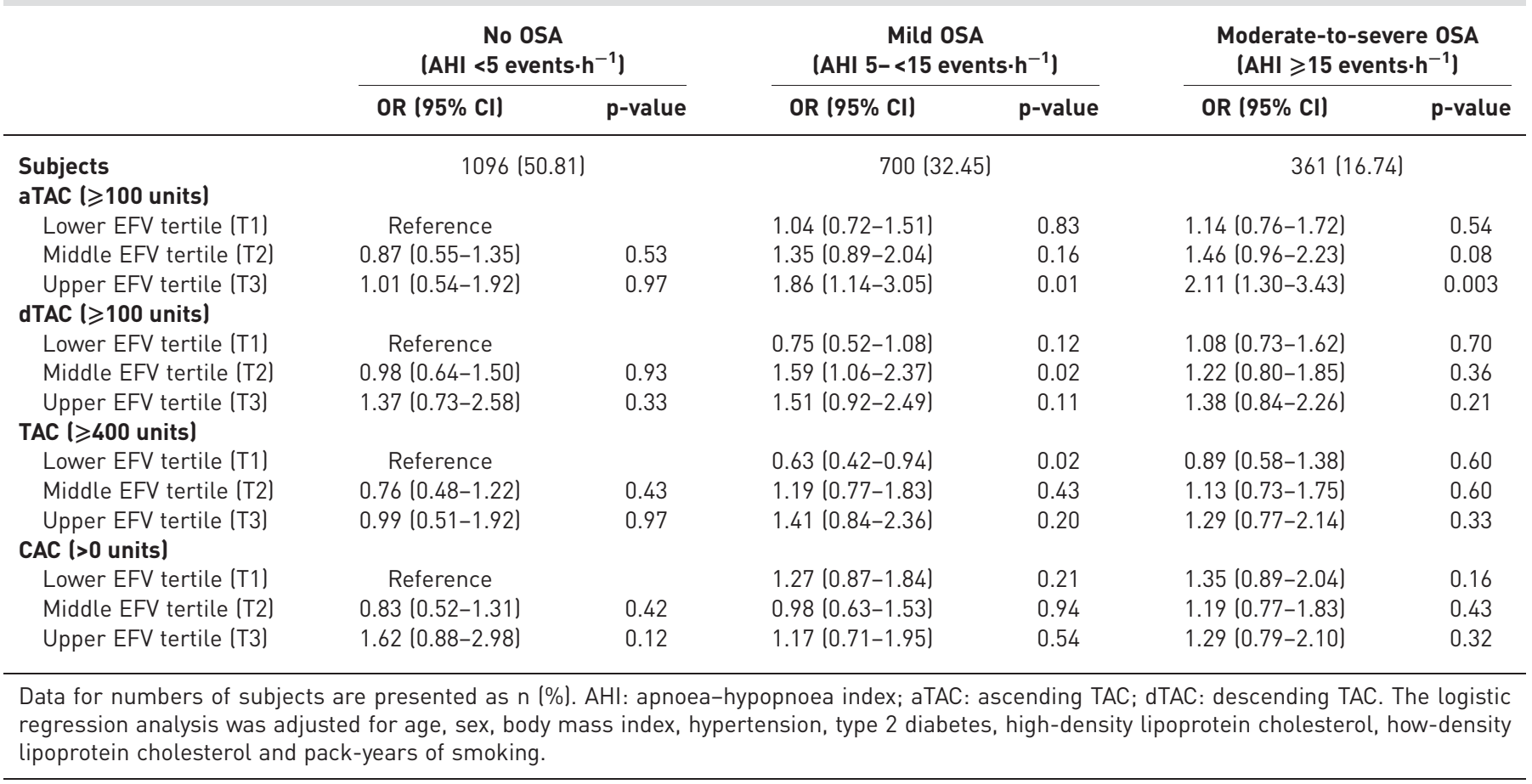


lowest EFV tertile (T1). However, participants with the highest EFV tertile (T3) were 1.01 times (95\% CI 0.54-1.92 times) more likely to have aTAC than participants with the lowest EFV tertile (T1) within those without OSA. The association between moderate-to-severe OSA and aTAC among participants in the highest EFV tertile (T3) was slightly stronger than in those without OSA and in the lowest EFV tertile (T1) after adjustment for cardiovascular risk factors (OR 2.11, 95\% CI 1.30-3.43; p=0.003). In addition, there was a moderate effect by EFV tertiles ( $p_{\text {interaction }}<0.001$ ) (figure 3 ).

\section{Discussion}

The principal finding of this study was that OSA severity, especially moderate-to-severe OSA, is independently associated with the presence of TAC, a useful predictor of subclinical systemic atherosclerosis in the general population without structural heart disease. In addition, we found an effect modification of EFV between OSA severity and TAC after adjustment for cardiovascular risk factors.

During non-rapid eye movement sleep, both the metabolic rate and the sympathetic nervous system activity normally decrease. However, sleep disordered breathing, such as OSA, interrupts this stationary phase by triggering a cascade of acute haemodynamic, inflammatory and metabolic effects, with chronic after-effects capable of initiating or exacerbating CVD [28]. Thus, repeated upper airway collapse and apnoeic spells result in arterial oxygen desaturation and arousal during sleep, leading to CVD such as blood pressure elevation and endothelial dysfunction [29]. The pathogenesis appears likely to be a multifactorial process involving a diverse range of mechanisms, including sympathetic nervous system overactivity, selective activation of inflammatory pathways, endothelial dysfunction and metabolic dysregulation [30], leading to increased arterial stiffness, arterial hypertension and the development of CVD. Several studies have reported the association between OSA and a higher incidence of CVD due to subclinical myocardial infarction or fatal CVD [15, 31]. In addition, endothelial dysfunction has been shown to develop in patients with OSA [32]. These trends have also been demonstrated in our study as the moderate-to-severe OSA group exhibited higher cardiovascular risk factors and a higher prevalence of hypertension and diabetes than the no OSA group.

Findings from studies determining the impact of OSA on vascular disease including the aorta have discussed several pathophysiological mechanisms, such as intrathoracic pressure swings leading to shear stress on artery walls, intermittent hypoxia leading to oxidative stress and sympathetic stimulation, and

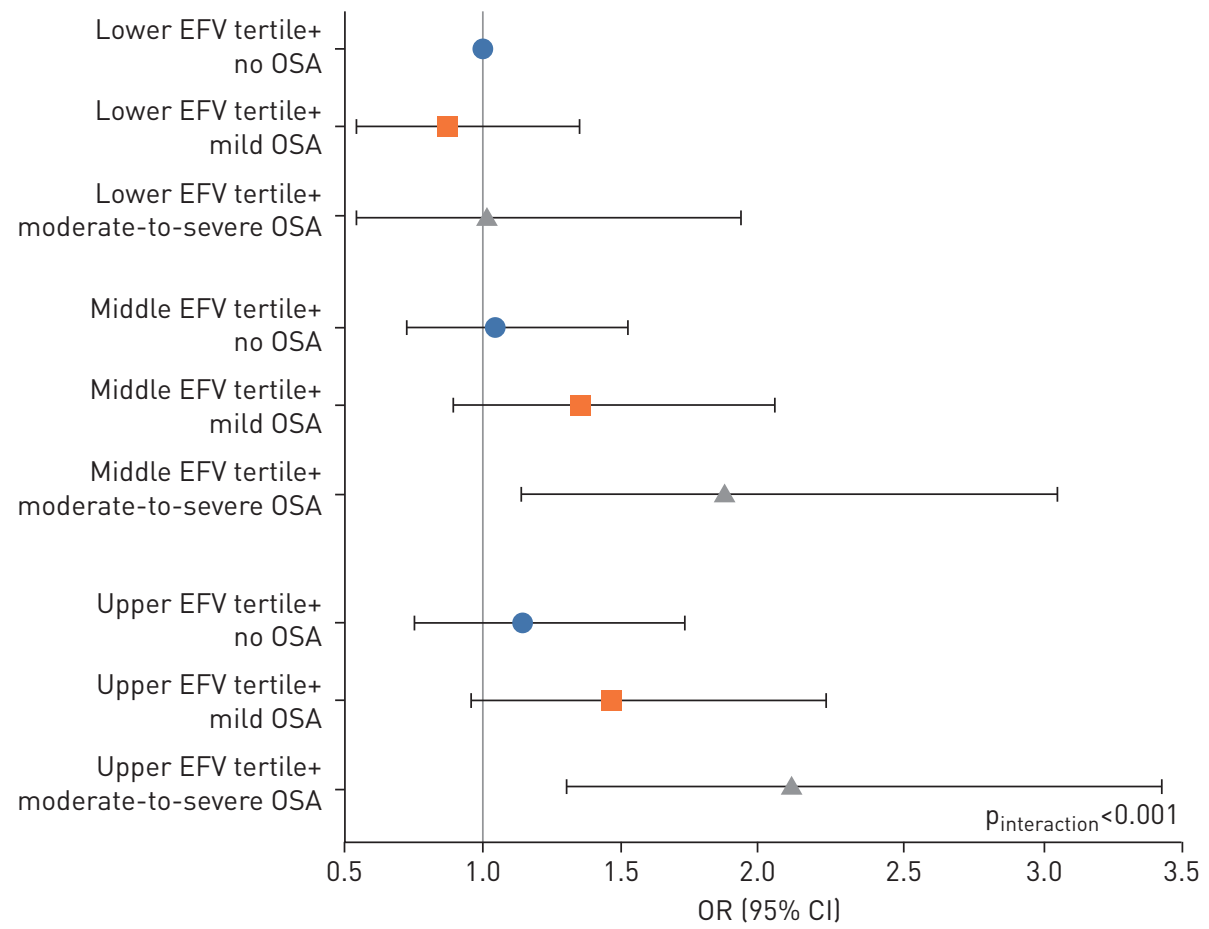

FIGURE 3 Joint effects of obstructive sleep apnoea (OSA) severity and epicardial fat volume (EFV) tertiles in the presence of ascending thoracic aorta calcification (aTAC $\geqslant 100$ units). Joint effects of OSA severity and EFV tertiles showed higher aTAC; the presence of aTAC was significantly higher in the upper EFV tertile (T3) group among participants with moderate-to-severe OSA than in the lower EFV tertile (T1) group among participants without OSA and this effect was dose-dependent on OSA severity. 
arousal-induced sympathetic activation with subsequent repetitive blood pressure elevations [2, 33]. The association between OSA and atherosclerotic diseases according to vascular calcification is highly suggestive, based on several epidemiological studies [13-17, 34, 35]. One study showed that subjects with severe OSA were more likely to have coronary atherosclerotic calcification (defined as CAC score $>0$ Agatston units) than those without OSA after adjusting for traditional cardiovascular risk factors [13], while less data are currently available regarding the association between OSA and aorta calcification. Recent evidence has indicated that OSA is associated with a greater extent of abdominal aortic calcification compared with no OSA [36].

Our study has demonstrated a significant relationship between moderate-to-severe OSA and TAC, but not with CAC. One possibility is that the impact of OSA on pathogenic pathways of developing atherosclerosis differs between CAC and TAC. TAC may represent systemic atherosclerosis burden especially better than CAC [37]. CAC is completely associated with atherosclerosis; however, TAC involves two pathophysiological processes: intimal, which is atherosclerotic, and medial, which contributes to increased aortic stiffness [37]. The potential impact of OSA on cardiovascular burden and aortic disease is likely to be related in large part to its association with elevated blood pressure. Previous cohort studies have consistently demonstrated that $>50 \%$ of subjects with OSA have hypertension [33], which has been revealed in our study as well (table 1). Hypertension more strongly influenced the aorta than the coronary artery [38] and aortic stiffness closely associated with aortic calcification is directly related to elevated systolic blood pressure [39]. It is generally accepted that hypertension can cause thoracic aortic dilation [40] and it is the main risk factor for aortic dissection [41], although the exact pathophysiology remains unclear. Unfortunately, although chest CT cannot differentiate between intimal and medial calcification, the contribution of medial calcification in TAC may be more significant for systemic atherosclerosis. In this study, the association with OSA severity and calcifications differed between the hypertensive and normotensive subgroups. In the normotensive group, OSA severity was not associated with any calcification scores. However, in the hypertensive group, OSA severity was associated with TACs up to 2.21 times higher than without OSA (supplementary table E1). It is therefore thought that OSA may act through other complex pathological mechanisms to elicit vascular damage.

Another possibility is that the thoracic aorta might be more vulnerable than the coronary arteries due to intrathoracic pressure swings related to OSA. The repetitive forced inspiration against the obstructed upper airway generates negative changes in intrathoracic pressure [33]. These forces lead to the vascular pressure stretching the aortic walls where blood pressure surges are highest and cause pathological shear stress. It is known that systolic blood pressure hastens the fragmentation of fibrin and collagen deposition with secondary stiffening of the aortic wall. Additional physical dilation or shear stress itself might be another important factor for developing atherosclerosis [2]. There is also the sequential development during life as aortic atherosclerosis is in general encountered earlier than coronary and carotid atherosclerosis [42]. In the present study, the presence of any TAC (including aTAC and dTAC) was already observed in individuals without CAC (supplementary figure E2). These results support the notion that OSA severity is more strongly related to TAC than CAC, especially in the general population without structural heart disease.

Obesity has been hypothesised to affect breathing by causing alterations in the upper airway structure or function, disturbances in the relationship between respiratory drive and load compensation, and exacerbations of sleep disordered breathing via obesity-related reductions in functional residual capacity and increased whole-body oxygen demand. EFV is well correlated with the presence of abdominal visceral adipose tissue [43]. In the present study, there was a positive correlation between EFV and body composition, including BMI and waist circumference (BMI: $\mathrm{r}=0.60,95 \%$ CI 0.57-0.62; waist circumference: $\mathrm{r}=0.67$, 95\% CI 0.65-0.69) (supplementary figure E1). Epicardial adipose tissue has been associated with the presence and severity of coronary artery disease in numerous studies [23, 44]. Furthermore, several case-control studies have investigated the relationship between epicardial adipose tissue and the presence and severity of OSA [45, 46], particularly in obese patients [1, 47]. Vascular calcification plays an important role in atherosclerosis, as well as in lipid accumulation and inflammation. In our previous preliminary study, both OSA and obesity were strongly associated with the presence and severity of a subclinical atherosclerotic burden, but the association between OSA and CAC became insignificant after further adjustment for BMI [16]. However, in this study, the presence of aTAC was higher in participants with moderate-to-severe OSA than in those without OSA, according to EFV tertiles. Moreover, the association between OSA and TAC was also significant after adjusting for EFV as well as BMI. Meanwhile, the presence of TAC was lower in mild OSA individuals (AHI $5-<15$ events $\cdot h^{-1}$ ) than in those without OSA among the lower EFV tertile group. The protective effects of low-frequency exposures to intermittent hypoxia can be explained by the activation of homeostatic or adaptive responses elicited by the intermittent hypoxia stimulus as preconditioning effects before the chronic pathophysiological process [48]. 
The strengths of this study are the inclusion of large general population-based samples, and the use of both objectively measured OSA severity by PSG and tomographic quantification of the calcium burden by noninvasive measurements. However, some limitations need to be considered when interpreting the results of our study. We used a cross-sectional study design; thus, a causal relationship needs to be determined in future studies.

In conclusion, our results support the finding that severity of OSA in general participants without structural heart disease is independently associated with subclinical systemic atherosclerosis. Multiple comorbid cardio-metabolic disorders of patients with OSA, especially in subjects with more epicardial fat, must be taken into consideration when investigating OSA-associated cardiovascular risk and systemic atherosclerosis. The challenges for future perspectives are in the development of specific preventive strategies targeting the pathways of cardiovascular calcification induced by severe intermittent hypoxia.

\section{Conflict of interest: None declared.}

Support statement: This study was supported by the Korea Centers for Disease Control and Prevention (KCDC) (2013-E71005-00, 2014-E71003-00, 2015-P71001-00 and 2016-E71003-00), the National Research Foundation of Korea (NRF) funded by the Korea government (MSIP) (NRF-2016R1A2B4012155, NRF-2017R1A6A3A11034663 and NRF-Brain Pool Program-2019H1D3A2A01101353), and the Korea University Grant. Funding information for this article has been deposited with the Crossref Funder Registry.

\section{References}

1 Mariani S, Fiore D, Barbaro G, et al. Association of epicardial fat thickness with the severity of obstructive sleep apnea in obese patients. Int J Cardiol 2013; 167: 2244-2249.

2 Gaisl T, Bratton DJ, Kohler M. The impact of obstructive sleep apnoea on the aorta. Eur Respir J 2015; 46 532-544.

3 Mirrakhimov AE, Sooronbaev T, Mirrakhimov EM. Prevalence of obstructive sleep apnea in Asian adults: a systematic review of the literature. BMC Pulm Med 2013; 13: 10.

4 Sunwoo JS, Hwangbo Y, Kim WJ, et al. Prevalence, sleep characteristics, and comorbidities in a population at high risk for obstructive sleep apnea: a nationwide questionnaire study in South Korea. PLoS One 2018; 13: e0193549.

5 Sia $\mathrm{CH}$, Hong Y, Tan LWL, et al. Awareness and knowledge of obstructive sleep apnea among the general population. Sleep Med 2017; 36: 10-17.

6 AlGhanim N, Comondore VR, Fleetham J, et al. The economic impact of obstructive sleep apnea. Lung 2008; 186: 7-12.

7 Lee W, Nagubadi S, Kryger MH, et al. Epidemiology of obstructive sleep apnea: a population-based perspective. Expert Rev Respir Med 2008; 2: 349-364.

8 Redline S, Yenokyan G, Gottlieb DJ, et al. Obstructive sleep apnea-hypopnea and incident stroke: the Sleep Heart Health Study. Am J Respir Crit Care Med 2010; 182: 269-277.

9 Marshall NS, Wong KK, Cullen SR, et al. Sleep apnea and 20-year follow-up for all-cause mortality, stroke, and cancer incidence and mortality in the Busselton Health Study cohort. J Clin Sleep Med 2014; 10: 355-362.

10 Drager LF, Polotsky VY, Lorenzi-Filho G. Obstructive sleep apnea: an emerging risk factor for atherosclerosis. Chest 2011; 140: 534-542.

11 Takasu J, Mao S, Budoff MJ. Aortic atherosclerosis detected with electron-beam CT as a predictor of obstructive coronary artery disease. Acad Radiol 2003; 10: 631-637.

12 Rumberger JA, Simons DB, Fitzpatrick LA, et al. Coronary artery calcium area by electron-beam computed tomography and coronary atherosclerotic plaque area. A histopathologic correlative study. Circulation 1995; 92: $2157-2162$.

13 Lutsey PL, McClelland RL, Duprez D, et al. Objectively measured sleep characteristics and prevalence of coronary artery calcification: the Multi-Ethnic Study of Atherosclerosis Sleep study. Thorax 2015; 70: 880-887.

14 Luyster FS, Kip KE, Aiyer AN, et al. Relation of obstructive sleep apnea to coronary artery calcium in non-obese versus obese men and women aged 45-75 years. Am J Cardiol 2014; 114: 1690-1694.

15 Seo MY, Lee JY, Hahn JY, et al. Association of obstructive sleep apnea with subclinical cardiovascular disease predicted by coronary artery calcium score in asymptomatic subjects. Am J Cardiol 2017; 120: 577-581.

16 Kim SH, Cho GY, Baik I, et al. Association of coronary artery calcification with obstructive sleep apnea and obesity in middle-aged men. Nutr Metab Cardiovasc Dis 2010; 20: 575-582.

17 Sorajja D, Gami AS, Somers VK, et al. Independent association between obstructive sleep apnea and subclinical coronary artery disease. Chest 2008; 133: 927-933.

18 Polak JF, Szklo M, Kronmal RA, et al. The value of carotid artery plaque and intima-media thickness for incident cardiovascular disease: the Multi-Ethnic Study of Atherosclerosis. J Am Heart Assoc 2013; 2: e000087.

19 Zhou M, Guo B, Wang Y, et al. The association between obstructive sleep apnea and carotid intima-media thickness: a systematic review and meta-analysis. Angiology 2017; 68: 575-583.

20 Shin C, Kim J, Kim J, et al. Association of habitual snoring with glucose and insulin metabolism in nonobese Korean adult men. Am J Respir Crit Care Med 2005; 171: 287-291.

21 Baik I, Kim J, Abbott RD, et al. Association of snoring with chronic bronchitis. Arch Intern Med 2008; 168: 167-173.

22 Berry RB, Budhiraja R, Gottlieb DJ, et al. Rules for scoring respiratory events in sleep: update of the 2007 AASM Manual for the Scoring of Sleep and Associated Events. Deliberations of the Sleep Apnea Definitions Task Force of the American Academy of Sleep Medicine. J Clin Sleep Med 2012; 8: 597-619.

23 Choo JY, Lee KY, Shin C, et al. Quantitative analysis of lungs and airways with CT in subjects with the chronic obstructive pulmonary disease (COPD) candidate FAM13A gene: case control study for CT quantification in COPD risk gene. J Comput Assist Tomogr 2014; 38: 597-603. 
24 Agatston AS, Janowitz WR, Hildner FJ, et al. Quantification of coronary artery calcium using ultrafast computed tomography. J Am Coll Cardiol 1990; 15: 827-832.

25 Wang TD, Lee WJ, Shih FY, et al. Association of epicardial adipose tissue with coronary atherosclerosis is region-specific and independent of conventional risk factors and intra-abdominal adiposity. Atherosclerosis 2010; 213: 279-287.

26 Kim J, Pack A, Maislin G, et al. Prospective observation on the association of snoring with subclinical changes in carotid atherosclerosis over four years. Sleep Med 2014; 15: 769-775.

27 Kim SH, Cho GY, Baik I, et al. Early abnormalities of cardiovascular structure and function in middle-aged Korean adults with prehypertension: The Korean Genome Epidemiology study. Am J Hypertens 2011; 24: $218-224$

28 Bradley TD, Floras JS. Obstructive sleep apnoea and its cardiovascular consequences. Lancet 2009; 373: 82-93.

29 Phillips BG, Narkiewicz K, Pesek CA, et al. Effects of obstructive sleep apnea on endothelin-1 and blood pressure. J Hypertens 1999; 17: 61-66.

30 McNicholas WT, Bonsigore MR, Management Committee of EU COST ACTION B26. Sleep apnoea as an independent risk factor for cardiovascular disease: current evidence, basic mechanisms and research priorities. Eur Respir J 2007; 29: 156-178.

31 Young T, Peppard PE, Gottlieb DJ. Epidemiology of obstructive sleep apnea: a population health perspective. Am J Respir Crit Care Med 2002; 165: 1217-1239.

32 Drager LF, Bortolotto LA, Lorenzi MC, et al. Early signs of atherosclerosis in obstructive sleep apnea. Am J Respir Crit Care Med 2005; 172: 613-618.

33 Kohler M, Stradling JR. Mechanisms of vascular damage in obstructive sleep apnea. Nat Rev Cardiol 2010; 7: 677-685.

34 Shahar E, Whitney CW, Redline S, et al. Sleep-disordered breathing and cardiovascular disease: cross-sectional results of the Sleep Heart Health Study. Am J Respir Crit Care Med 2001; 163: 19-25.

35 Peker Y, Kraiczi H, Hedner J, et al. An independent association between obstructive sleep apnoea and coronary artery disease. Eur Respir J 1999; 14: 179-184.

36 Tachikawa R, Koyasu S, Matsumoto T, et al. Obstructive sleep apnea and abdominal aortic calcification: is there an association independent of comorbid risk factors? Atherosclerosis 2015; 241: 6-11.

37 Budoff MJ, Nasir K, Katz R, et al. Thoracic aortic calcification and coronary heart disease events: the Multi-Ethnic Study of Atherosclerosis (MESA). Atherosclerosis 2011; 215: 196-202.

38 Takasu J, Budoff MJ, O’Brien KD, et al. Relationship between coronary artery and descending thoracic aortic calcification as detected by computed tomography: the Multi-Ethnic Study of Atherosclerosis. Atherosclerosis 2009; 204: 440-446.

39 O'Rourke MF, Nichols WW. Aortic diameter, aortic stiffness, and wave reflection increase with age and isolated systolic hypertension. Hypertension 2005; 45: 652-658.

40 Vasan RS, Larson MG, Levy D. Determinants of echocardiographic aortic root size. The Framingham Heart Study. Circulation 1995; 91: 734-740.

41 Hagan PG, Nienaber CA, Isselbacher EM, et al. The International Registry of Acute Aortic Dissection (IRAD): new insights into an old disease. JAMA 2000; 283: 897-903.

42 Shah AM, Banerjee T, Mukherjee D. Coronary, peripheral and cerebrovascular disease: a complex relationship. Herz 2008; 33: 475-480.

43 Iacobellis G, Ribaudo MC, Assael F, et al. Echocardiographic epicardial adipose tissue is related to anthropometric and clinical parameters of metabolic syndrome: a new indicator of cardiovascular risk. J Clin Endocrinol Metab 2003; 88: 5163-5168.

44 Bachar GN, Dicker D, Kornowski R, et al. Epicardial adipose tissue as a predictor of coronary artery disease in asymptomatic subjects. Am J Cardiol 2012; 110: 534-538.

45 Akilli H, Kayrak M, Bekci TT, et al. Gender-related changes of the epicardial fat thickness and leptin in obstructive sleep apnea. Echocardiography 2014; 31: 411-419.

46 Kostopoulos K, Alhanatis E, Pampoukas K, et al. CPAP therapy induces favorable short-term changes in epicardial fat thickness and vascular and metabolic markers in apparently healthy subjects with obstructive sleep apnea-hypopnea syndrome (OSAHS). Sleep Breath 2016; 20: 483-493.

47 Lubrano C, Saponara M, Barbaro G, et al. Relationships between body fat distribution, epicardial fat and obstructive sleep apnea in obese patients with and without metabolic syndrome. PLoS One 2012; 7: e47059.

48 Almendros I, Wang Y, Gozal D. The polymorphic and contradictory aspects of intermittent hypoxia. Am J Physiol Lung Cell Mol Physiol 2014; 307: L129-L140. 